\title{
Effect of providing information about normal test results on patients' reassurance: randomised controlled trial
}

\author{
Keith J Petrie, professor, ${ }^{1}$ Jan Tobias Müller, diplom psychologist, ${ }^{3}$ Frederike Schirmbeck, diplom \\ psychologist, ${ }^{3}$ Liesje Donkin, student, ${ }^{1}$ Elizabeth Broadbent, lecturer, ${ }^{1}$ Christopher J Ellis, cardiologist, ${ }^{2}$ \\ Greg Gamble, statistician, ${ }^{2}$ Winfried Rief, professor ${ }^{3}$
}

Department of Psychological Medicine, Faculty of Medical and Health Sciences, University of Auckland, Private Bag 92019, New Zealand

${ }^{2}$ Department of Cardiology, Auckland City Hospital

${ }^{3}$ Philipps-University, Faculty of Psychology, Marburg, Germany

Correspondence to:

KJ Petrie

kj.petrie@auckland.ac.nz

doi: 10.1136/bmj.39093.464190.55

\section{ABSTRACT}

Objective To investigate whether providing information about normal findings before a diagnostic test improves patients' reassurance and reduces anxiety about symptoms.

Design Randomised controlled trial.

Setting Outpatient cardiology clinic.

Participants 92 patients with chest pain referred for a diagnostic exercise stress test.

Intervention Before undergoing testing patients were randomised to receive standard information $(n=28$; control group), a pamphlet explaining the function of the test and the meaning of normal test results $(n=30$; pamphlet group), or the pamphlet and a brief discussion about the meaning of normal test results $(n=34$; discussion group).

Main outcome measures The primary outcome was patients' reported reassurance on a 5 item scale immediately after the test and at one month. Secondary outcomes were the proportion of patients still with chest pain and still taking cardiac drugs at one month.

Results The mean levels of reassurance after testing and feedback from the doctor were significantly higher in the discussion group (42.0, $95 \%$ confidence interval 39.7 to 44.2) than in the pamphlet $(39.2,36.1$ to 42.3$)$ and control groups (35.8, 31.6 to 39.9$)$. This difference was maintained at one month. The proportion of patients still reporting chest pain at one month decreased significantly in the discussion group (to 17\%) and pamphlet group (to $28 \%$ ) but not in the control group (to $36 \%$ ). A trend was for fewer patients in the discussion group to be taking cardiac drugs at one month.

Conclusion Providing patients with information about normal test results before testing can improve rates of reassurance and reduce the likelihood of future reports of chest pain.

Trial registration Current Controlled Trials ISRCTN87589121.

\section{INTRODUCTION}

Reassurance is one of the most common interventions in medical practice. Yet many patients with symptoms remain anxious about their condition even after investigations and reassurance from their doctor. ${ }^{12}$ Such patients often continue to be disabled by their condition, use drugs inappropriately, and seek medical help from other health professionals for their symptoms. $^{34}$

Doctors typically give reassurance after investigations. Although this may seem logical, evidence suggests that by the time patients undergo tests many have already developed negative ideas and beliefs about their symptoms, and thus reassurance is much less effective. ${ }^{5}$ This may especially be the case when there are delays in completing investigations. Patients' established negative beliefs about their symptoms may limit their ability to assimilate reassuring messages that are by then inconsistent with their view of the seriousness of their condition. ${ }^{6}$ Furthermore, increasing the amount of reassurance after investigations does not always seem to reduce concerns about symptoms. ${ }^{7}$

The effects that patients' pre-existing ideas have on reassurance suggest a possible pathway to improve reassurance. Providing an explanation about the meaning of normal test results before testing may weaken patients' preconceived ideas about their illness and provide a context to help patients make sense of the test result. Patients will be better prepared to receive reassurance from their doctor and the effects will be strengthened.

We investigated whether giving patients information about a diagnostic test and discussing the meaning of normal results before the test would improve rates of reassurance.

\section{METHODS}

Eligible participants were those with chest pain referred for a diagnostic exercise stress test at Auckland City Hospital. The study took place between June and October 2004. We excluded patients aged less than 18 years and those who had a previous diagnosis of cardiac disease, had no symptoms of chest pain, or were undergoing the test as part of a presurgical examination. Participants were randomised to one of three intervention groups according to a computer generated random number sequence. Allocation was concealed in sequentially numbered sealed opaque envelopes. 


\section{Intervention groups}

\section{Control group}

Patients in the control group received a sheet of information on the exercise stress test from the cardiology clinic with their clinic appointment. This included advice on what to wear, the procedure, and the risks of complications.

\section{Pamphlet group}

The pamphlet group received a 450 word pamphlet to read before their stress exercise test (additional information is provided by the authors at www.health.auckland.ac.nz/psych-med/staff/keiths $\% 20$ papers/bmj $\%$ 20appendix.html). The pamphlet explained the function of the test, the meaning of normal results, and other possible reasons for chest pain that were less serious. The pamphlet was divided into seven sections, with each section headed by the following questions: What is an exercise stress test? How does the exercise stress test work? What happens during testing? What if it is too hard for me? What could the results mean? Could there be other causes for my chest pain? What if my chest pain continues but my test is normal?

\section{Discussion group}

Patients in the discussion group received the pamphlet and were later engaged in discussion with the research health psychologist, who asked if they had any questions about what they had read. The psychologist briefly reiterated the main points of the pamphletthat a lot of people with chest pain worry that there might be something wrong with their heart; that if the result of the test is normal the patient's risk for coronary artery disease is as low as for anyone in the general population; and that just because the pain may not be related to the heart does not mean that it is not real pain and that it is important to keep in mind that many other causes of chest pain are less serious.

Patients completed the test according to the standard Bruce exercise stress testing protocol. ${ }^{8}$ When results were negative, a cardiology registrar provided patients with their standard reassurance that the result was normal and did not show cardiac disease and that a report would be sent to their general practitioner.

\section{Baseline assessment}

Patients meeting the eligibility criteria were approached in the waiting room. After providing informed consent they completed a questionnaire on personal data, pain ratings, concerns about symptoms, and self-rated health. They were asked to rate how worried they were about their health (from 0 "not at all" to 10 "extremely") and the extent to which they believed there was something seriously wrong with their heart (from 0 "not at all" to 10 "strongly believe"). The psychologist then opened the envelope with the randomisation code.

\section{Post-testing questionnaire}

Immediately after the test the patients completed a brief questionnaire comprising the two items on concerns about their health and heart and three further items. They were asked to rate on 10 point scales the extent to which they were reassured by the test, the extent to which they believed they needed further tests to determine the cause of their illness, and how accurate they thought the test for identifying heart problems. After reversing three of the negatively worded items we summed the scores for these five items to create a scale for reassurance, with higher scores indicating higher levels of reassurance. These items for reassurance have been used previously, ${ }^{5}$ and the scale showed acceptable internal consistency (Cronbach's $\alpha=0.80)$.

\section{One month follow-up}

At one month a researcher blind to the allocation group telephoned the patients and completed the reassurance scale. Patients were also asked if they still had chest pain and if they were still taking cardiac drugs.

\section{Statistical analysis}

We defined participants as reassured when their reassurance level was above the median for the total sample. Our study is thus conservatively powered to detect a medium (Cohen $\mathrm{W}=0.38$ ) effect size using PASS. ${ }^{9}$ This effect size is achieved with a sample size of 66 (80\% power, 5\% significance level) split between three groups. We therefore recruited 90 participants to compensate for losses to follow-up.

We used SAS statistical software v 9.1 for the analyses. We brought post-test data forward as a proxy for the assessment at one month of the five participants lost to follow-up. To examine differences between the groups on the reassurance scale we used a repeated measures mixed model analysis. We used Tukey post-hoc tests to determine differences between individual groups and across time. To determine potential differences in the proportion of patients reassured (above median at post-testing and follow-up) between the groups and over time we used a categorical modelling procedure (SAS Proc Catmod). McNemar's test was used to assess whether there were within group differences in reported chest pain. All tests were two tailed and we considered $\mathrm{P}<0.05$ as significant.

\section{RESULTS}

Of 97 eligible adults with chest pain referred for a diagnostic exercise stress test, five declined to participate (fig 1). Overall, 28 were randomised to receive standard information on the test, 30 to receive an information pamphlet and explanation of the meaning of normal test results, and 34 to receive the pamphlet and a brief discussion about the meaning of normal test results. Fifteen had a positive test result and were excluded. All patients completed the brief post-test assessment. Five patients could not be traced at one month.

Table 1 lists the baseline personal characteristics and clinical details of the experimental groups, including waiting times for the investigation, experience of a previous exercise stress test, and pain. The groups were 


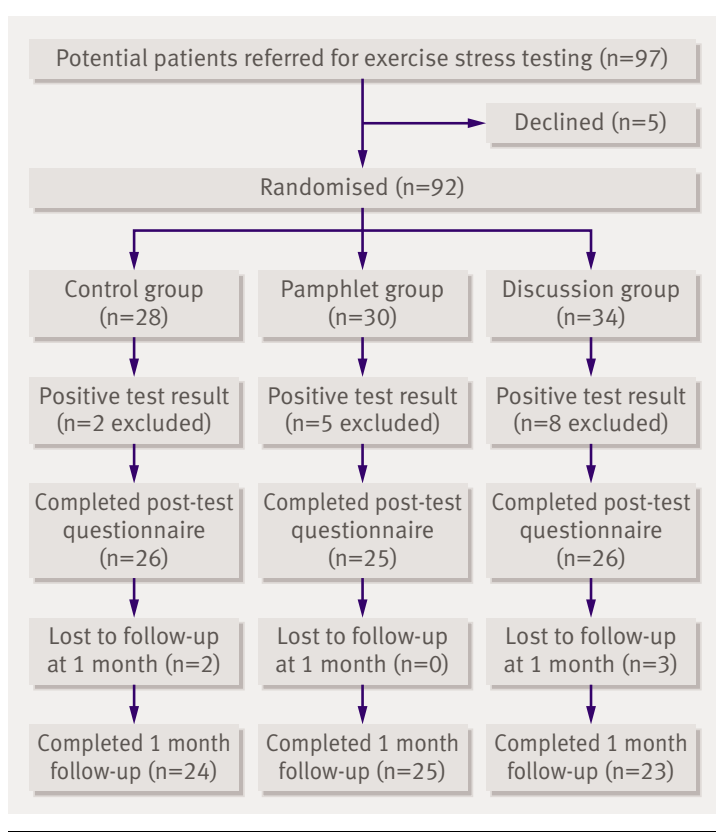

Fig 1| Participant flow through study

Table 1| Personal and clinical characteristics of groups at baseline. Values are numbers (percentages) unless stated otherwise

\begin{tabular}{|c|c|c|c|}
\hline Variable & $\begin{array}{l}\text { Control } \\
\text { group } \\
(n=28)\end{array}$ & $\begin{array}{c}\text { Pamphlet } \\
\text { group }(n=30)\end{array}$ & $\begin{array}{l}\text { Discussion } \\
\text { group } \\
(n=34)\end{array}$ \\
\hline Mean (SD) age (years) & $52.2(13.2)$ & $56.9(13.6)$ & $52.2(13.3)$ \\
\hline Men & 19 & 16 & 17 \\
\hline Women & 9 & 14 & 17 \\
\hline \multicolumn{4}{|l|}{ Ethnicity: } \\
\hline European & 18 & 22 & 23 \\
\hline Other & 10 & 8 & 11 \\
\hline \multicolumn{4}{|l|}{ Education: } \\
\hline Secondary & 16 & 15 & 19 \\
\hline Post & 12 & 15 & 15 \\
\hline \multicolumn{4}{|l|}{ Employment: } \\
\hline Full time & 14 & 13 & 20 \\
\hline Other & 14 & 17 & 14 \\
\hline \multicolumn{4}{|l|}{ Previous stress test: } \\
\hline Yes & 4 & 9 & 8 \\
\hline No & 24 & 21 & 26 \\
\hline $\begin{array}{l}\text { Mean (SD wait for } \\
\text { investigation (weeks) }\end{array}$ & $8.1(10.0)$ & $6.7(6.7)$ & $8.4(10.4)$ \\
\hline $\begin{array}{l}\text { Mean (SD) pain severity } \\
(1-10)^{\star}\end{array}$ & $3.2(2.2)$ & $3.4(2.1)$ & $3.2(2.4)$ \\
\hline $\begin{array}{l}\text { Mean (SD) pain } \\
\text { limitation }(1-10) \dagger\end{array}$ & $2.2(2.4)$ & $2.6(2.2)$ & $2.1(2.0)$ \\
\hline $\begin{array}{l}\text { Mean (SD) score for "How } \\
\text { worried are you about } \\
\text { your health?" }(1-10) \ddagger\end{array}$ & $4.4(2.9)$ & $6.2(2.7)$ & $5.4(2.5)$ \\
\hline $\begin{array}{l}\text { Mean (SD) score for "Do } \\
\text { you believe something is } \\
\text { seriously wrong with your } \\
\text { heart?" (1-10)§ }\end{array}$ & $7.4(2.5)$ & $8.6(1.5)$ & $7.3(2.2)$ \\
\hline \multicolumn{4}{|c|}{$\begin{array}{l}\star 1=\text { no pain; } 10=\text { severe pain. } \\
\dagger 1=\text { not at all; } 10=\text { extremely limited. } \\
\ddagger 1=\text { not at all; } 10=\text { extremely. } \\
\S 1=\text { not at all; } 10=\text { strongly believe. }\end{array}$} \\
\hline
\end{tabular}

well balanced. The repeated measures analysis showed a significant difference between the groups on the reassurance scale after the test and at the one month followup (table $2 ; \mathrm{P}=0.002$ ) and no interaction with time $(\mathrm{P}=0.25)$. A retrospective analysis showed a significantly higher level of reassurance in the discussion group than in the control group.

Significant differences were found between the groups in reassurance after testing and at one month follow-up $\left(\chi^{2}=7, \mathrm{df}=2, \mathrm{P}=0.03\right)$. In the retrospective analysis the discussion group had a higher proportion of reassured patients $(65 \%)$ after testing than the control $(50 \%)$ and pamphlet $(44 \%)$ groups $(\mathrm{P}=0.03$ and $\mathrm{P}=0.02)$. At one month this difference was maintained $\left(\chi^{2}=1.92, \mathrm{df}=2, \mathrm{P}=0.38\right)$, with $69 \%$ of patients still reassured in the discussion group compared with $40 \%$ in the pamphlet group and 35\% in the control group. No significant difference was found between the control and pamphlet groups $(\mathrm{P}=0.99)$. More participants in the control and pamphlet groups had lower levels of reassurance at both time points than those in the discussion group (fig 2).

All patients needed to have symptoms of chest pain to be included in the trial. At one month the numbers of patients who mentioned chest pain had reduced significantly to $4(17 \%)$ in the discussion group $(\mathrm{P}<0.001)$ and to $7(28 \%)$ in the pamphlet group $(\mathrm{P}=0.005)$ but the reduction in the control group to $9(36 \%)$ was not significant $(\mathrm{P}=0.09)$. Consistent with these findings was a trend for fewer patients in the discussion group to be taking cardiac drugs: $6(25 \%)$ patients in the control group, $2(8 \%)$ in the pamphlet group, and $1(4.3 \%)$ in the discussion group $\left(\chi^{2}=5.3, \mathrm{df}=2, \mathrm{P}=0.07\right)$.

\begin{tabular}{|c|c|c|}
\hline \multicolumn{3}{|c|}{$\begin{array}{l}\text { Table } 2 \mid \text { Reassurance scores after exercise stress test and at } \\
\text { one month follow-up in groups }\end{array}$} \\
\hline Group & $\begin{array}{l}\text { Mean }(95 \% \mathrm{Cl}) \text { post- } \\
\text { test scores }\end{array}$ & $\begin{array}{l}\text { Mean }(95 \% \mathrm{Cl}) \text { follow-up } \\
\text { scores }\end{array}$ \\
\hline Control & 35.8 (31.6 to 39.9) & 34.4 (30.5 to 38.4$)$ \\
\hline Pamphlet & $39.2(36.1$ to 42.3$)$ & $38.4(35.4$ to 41.4$)$ \\
\hline Discussion & 42.0 (39.7 to 44.2$)$ & $43.4(41.0$ to 45.8$)$ \\
\hline
\end{tabular}

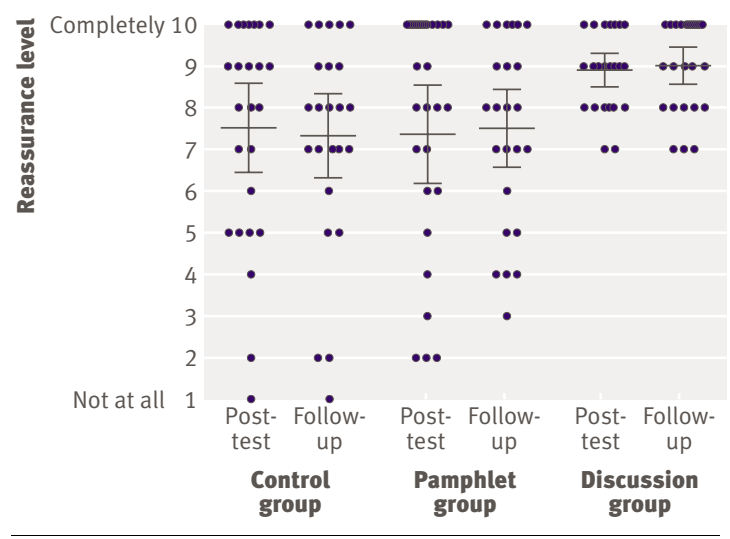

Fig 2 | Dot plot for item asking patients how reassured they were by the exercise stress test after testing and at one month follow-up in experimental groups, including means ( $95 \%$ confidence intervals) 


\section{WHAT IS ALREADY KNOWN ON THIS TOPIC}

Reassurance from doctors is a common medical intervention

Even after the completion of investigations and reassurance from doctors, many patients remain anxious about their symptoms

\section{WHAT THIS STUDY ADDS}

Written information and a discussion about normal results before testing improved rates of patients' reassurance follow-up period, which would enable the assessment of any differences in future medical investigations in the study groups and an estimate of the economic benefits of the intervention. We used a health psychologist to engage patients in a discussion about the test and it remains to be established whether similar results can be obtained with a clinic nurse or registrar.

This relatively small study may best be considered as a proof of principle study, the results of which need replication in a larger sample. The implication of the study for clinicians is that an increase in patients' reassurance after clinical testing can be expected if more time is spent explaining the meaning of normal test results before the test. Improvements in reassurance are also likely to impact on unnecessary future investigations and patients' anxieties about their symptoms.

Contributors: KJP conceived and designed the study, drafted the manuscript, and is guarantor. JTM, FS, and LD helped develop the study materials and run the trial. GG, EB, CIE, and WR helped with data interpretation. CJE, WR, EB, and GG helped draft the paper. All authors contributed to and approved the final version.

Funding: University of Auckland.

Competing interests: None declared.

Ethical approval: This study was approved by the New Zealand Ministry of Health Ethics Committee (AKY/04/05/121).

1 Howard L, Wessely S. Reappraising reassurance: the role of investigations. J Psychosom Res 1996;41:307-11.

2 McDonald IG, Daly J, Jelinek VM, Panetta F, Gutman JM. Opening Pandora's box: the unpredictability of reassurance by a normal test result. $B M J$ 1996:313:329-32.

3 Potts SG, Bass CM. Psychological morbidity in patients with chest pain and normal or near normal coronary arteries: a long-term followup study. Psychol Med 1995;25:339-47.

4 Fitzpatrick R, Hopkins A. Referrals to neurologists for headaches not due to structural disease. J Neurol Neurosurg Psychiatry 1981:44:1061-7.

5 Donkin L, Ellis C], Powell R, Broadbent E, Gamble G, Petrie KJ. Illness perceptions predict reassurance following negative exercise testing result. Psychol Health 2006;21:421-30.

6 Nijher G, Weinman J, Bass C, Chambers J. Chest pain in people with normal coronary arteries. BMJ 2001;323:1319-20.

7 Sanders D, Bass C, Mayou RA, Goodwin S, Bryant BM, Tyndel S. Noncardiac chest pain: why was a brief intervention apparently ineffective? Psychol Med 1997;27:1033-40.

8 Bruce RA, Hossack KF, DeRouen TA, Hofer V. Enhanced risk assessment for primary coronary heart disease events by maximal exercise testing: 10 years' experience of Seattle Heart Watch. J Am Coll Cardiol 1983;2:565-73.

9 Hintze J. NCSS and PASS software. Kaysville, UT: Number Cruncher Statistical Systems, 2004.

10 Lucock MP, Morley S, White C, Peake MD. Responses of consecutive patients to reassurance after gastroscopy: results of a self administered questionnaire survey. BMJ 1997;315:572-5.

11 Channer KS, James MA, Papouchado M, Rees JR. Failure of a negative exercise test to reassure patients with chest pain. QJ Med 1987;63:315-22.

12 Meechan GT, Collins JP, Moss-Morris R, Petrie KJ. Who is not reassured following benign diagnosis of breast symptoms? Psychooncol 2005;14:239-46.

Accepted: 12 December 2006 